\title{
Small-cell lung carcinoma transformation of lung adenocarcinoma diagnosed by pericardial effusion: A case report
}

\author{
RYOTA OTOSHI $^{1}$, AKIMASA SEKINE ${ }^{1}$, KOJI OKUDELA $^{2}$, MASATO ASAOKA $^{1}$, YOZO SATO ${ }^{1}$, \\ SATOSHI IKEDA ${ }^{1}$, TOMOHISA BABA ${ }^{1}$, SHIGERU KOMATSU ${ }^{1}$, ERI HAGIWARA ${ }^{1}$ and TAKASHI OGURA ${ }^{1}$ \\ ${ }^{1}$ Department of Respiratory Medicine, Kanagawa Cardiovascular and Respiratory Center, Yokohama, Kanagawa 236-0051; \\ ${ }^{2}$ Department of Pathology, Yokohama City University Graduate School of Medicine, Yokohama, Kanagawa 236-0027, Japan
}

Received August 22, 2019; Accepted April 30, 2020

DOI: $10.3892 / \mathrm{mco} .2020 .2059$

\begin{abstract}
The present report describes a case of a 68-year-old male patient with epidermal growth factor receptor (EGFR)-mutant non-small cell lung carcinoma (NSCLC). After cytotoxic chemotherapy of three regimens following 22 months of treatment with EGFR-tyrosine kinase inhibitors (EGFR-TKIs), including osimertinib, the patient underwent S-1 treatment. Despite a decrease in carcinoembryonic antigen 1 month after initiating S-1 treatment, the patient developed cardiac tamponade. The evaluation of pericardial effusion confirmed small-cell lung carcinoma (SCLC) transformation. Subsequently, a combination therapy of carboplatin and etoposide was administered, which led to a marked improvement in imaging. In patients with NSCLC who develop pericardial effusion after long-term EGFR-TKI therapy, including osimertinib treatment, it is important to investigate whether SCLC transformation occurs or not as a treatable entity.
\end{abstract}

\section{Introduction}

Non-small-cell lung carcinoma (NSCLC) with epidermal growth factor receptor (EGFR) mutations responds well to epidermal growth factor receptor tyrosine kinase inhibitors (EGFR-TKIs). However, most patients acquire resistance to EGFR-TKIs and experience disease progression (1). Although several resistance mechanisms to EGFR-TKIs have been recently reported, small-cell lung carcinoma (SCLC) transformation is a relatively rare resistance mechanism and mostly

Correspondence to: Dr Akimasa Sekine, Department of Respiratory Medicine, Kanagawa Cardiovascular and Respiratory Center, 6-16-1 Tomioka-higashi, Kanazawa-ku, Yokohama, Kanagawa 236-0051, Japan

E-mail: akimasa.sekine@gmail.com

Key words: small-cell lung carcinoma transformation, epidermal growth factor receptor, osimertinib, adenocarcinoma, pericardial effusion develops in NSCLC patients with 1st and 2nd generation EGFR-TKIs such as gefitinib, erlotinib, and afatinib $(2,3)$. To date, only a few cases of SCLC transformation during or after treatment with osimertinib have been reported (4-8). However, all previously reported cases of SCLC transformation have been diagnosed by pathological tissues of primary lesion or metastatic lesions, not pericardial effusion. Here, we report the case of malignant pericarditis developed in SCLC transformed NSCLC patient with a history of osimertinib treatment.

\section{Case report}

A 68-year-old man with a smoking history of 40 packs per year was admitted to our hospital due to relapse of stage IA lung adenocarcinoma in December 2014, which was previously surgically resected in December 2012. Computed tomography $(\mathrm{CT})$ revealed mediastinal lymphadenopathy and multiple lung nodules (Fig. 1). In addition, serum carcinoembryonic antigen (CEA) level had increased to $16.0 \mathrm{ng} / \mathrm{ml}$. The specimen obtained by endobronchial ultrasound-guided transbronchial needle aspiration to mediastinal lymph node (\#4R) confirmed lung adenocarcinoma with an EGFR mutation (exon 19 deletion) by therascreen ${ }^{\circledR}$ EGFR RGQ PCR Kit (Qiagen, Hilden, Germany). Then, he was enrolled in a clinical trial (FLAURA trial) comparing osimertinib and gefitinib (or erlotinib) as the 1st line chemotherapy and treated with osimertinib in March 2015 (9). Although this treatment had a significant response lasting 11 months and CEA fell to the normal level, routine-follow-up CT in February 2016 showed slight ground-glass attenuation (GGA) in the right lower lobe. The treatment was discontinued on suspicion of drug-induced pneumonitis. Although GGA was spontaneously regressed, mediastinal lymphadenopathy and multiple lung nodules aggravated. Therefore, erlotinib treatment as 2nd line chemotherapy was initiated with a careful observation in May 2016. After 8 months treatment with erlotinib, follow-up CT in January 2017 demonstrated re-progression of the mediastinal lymph node and appearance of minor amounts of pericardial effusion. With a re-biopsy of the lymph node (\#4R), lung adenocarcinoma was found the EGFR T790M mutation in addition to the EGFR exon 19 deletion although liquid biopsy, 


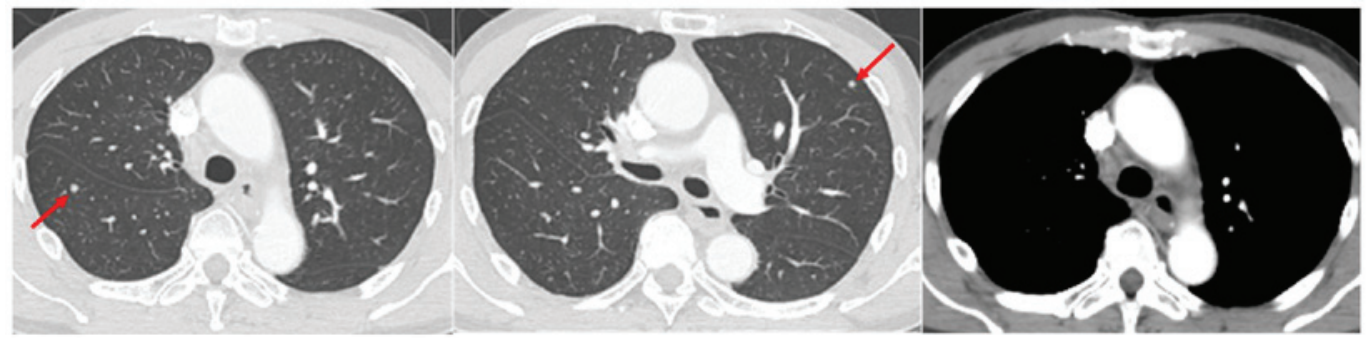

Figure 1. Chest computed tomography revealed multiple lung nodules (arrow) and mediastinal lymphadenopathy, including right lower paratrachel lymph node, at relapse.

A

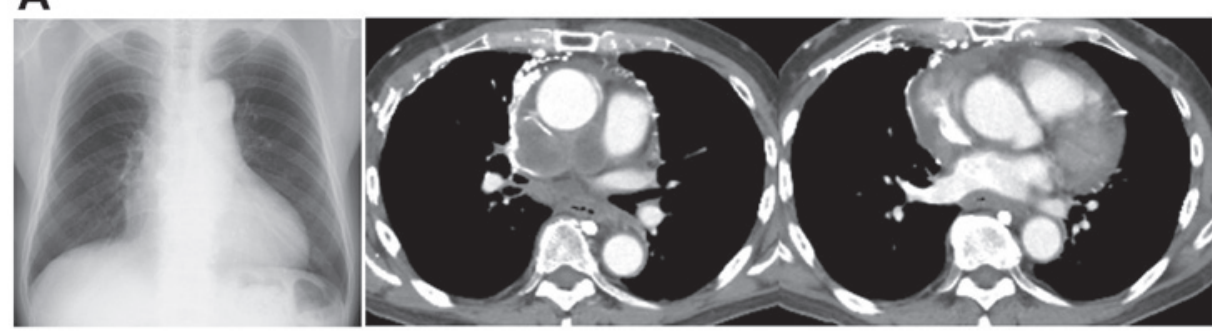

B

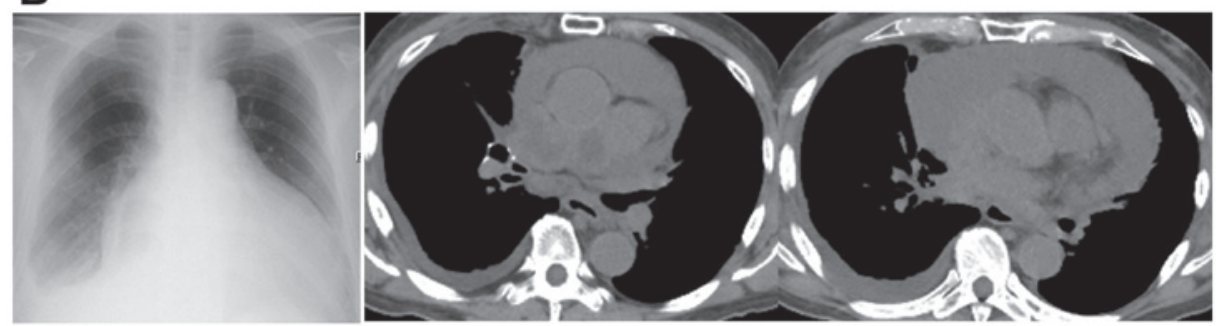

C

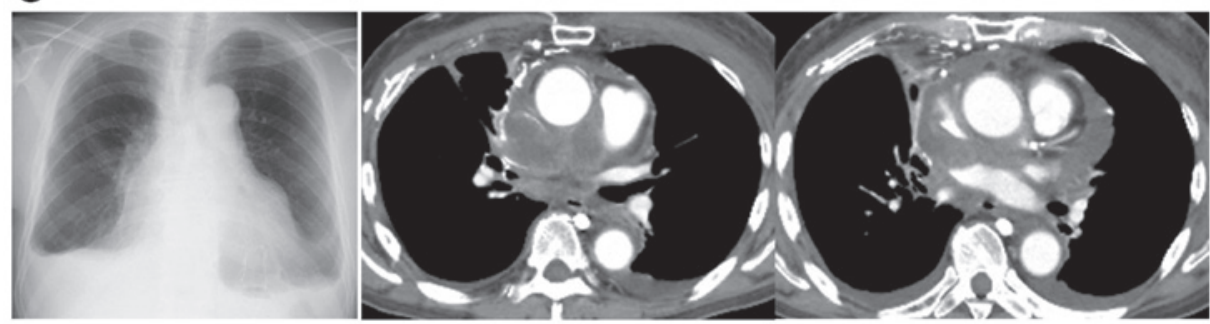

D

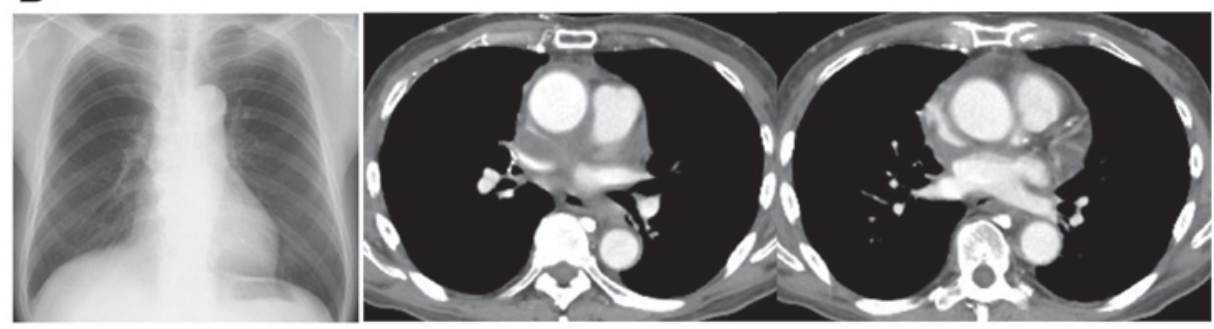

Figure 2. Changes in imaging of the patient over time. (A) Before treatment with S-1, a chest radiograph revealed mild enlargement of the cardiac silhouette. Chest computed tomography demonstrated mediastinal tumor and minor amount of pericardial effusion. (B) Chest radiograph showing apparent enlargement of the cardiac silhouette and pleural effusion. Chest computed tomography revealed apparent enlargement of mediastinal tumors and an increase in pericardial effusion. (C) After drainage, pericardial effusion decreased but was still present. (D) Mediastinal tumor and pericardial effusion markedly decreased after combination therapy of carboplatin and etoposide was initiated.

a blood test that detects evidence of cancer cells or tumor DNA, showed only exon 19 deletion by cobas EGFR Mutation test v2 (Roche Molecular Systems, Pleasanton, CA, USA). Thus, re-challenge with osimertinib as 3rd line treatment was initiated, and three month later, despite a tentative response, enlargement of mediastinal tumors with an elevated CEA level of $24.3 \mathrm{ng} / \mathrm{ml}$ was observed. Next, the patient was treated with carboplatin in combination with paclitaxel, docetaxel, and 


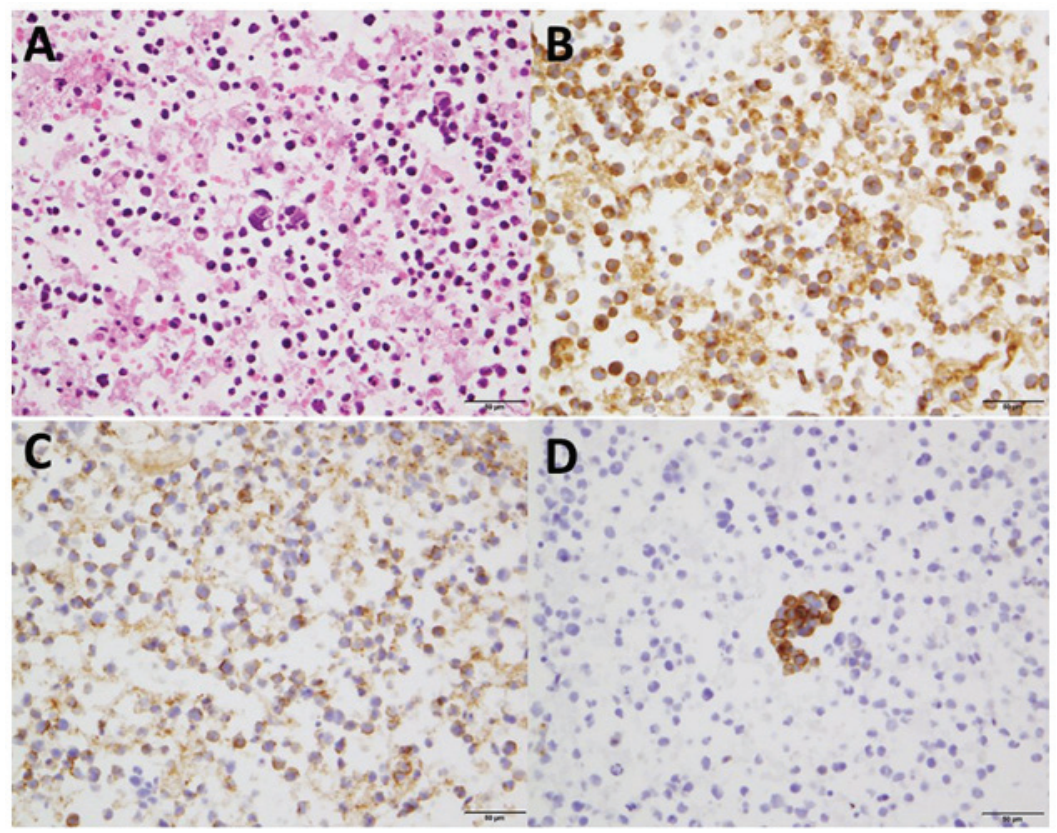

Figure 3. Histological findings of the cell block from pericardial effusion. The specimens showed poorly differentiated cells with a high nuclear-to-cytoplasmic ratio. (A) Hematoxylin and eosin staining. Magnification, x400. Immunostaining revealed that (B) synaptophysin, (C) NCAM and (D) chromogranin were positive. Magnification, $\mathrm{x} 400$.

pemetrexed as 4th-6th line treatment, respectively, which did not present a desired effect and CEA level remained elevated. Thus, S-1 monotherapy was initiated in August 2018 as 7th line treatment (Fig. 2A). One month after this treatment was initiated, an apparent decrease in CEA level from $100.5 \mathrm{ng} / \mathrm{ml}$ to $30 \mathrm{ng} / \mathrm{ml}$ was observed. However, dyspnea gradually appeared, and CT showed apparent enlargement of mediastinal tumors and increase of pericardial effusion, which ultimately resulted in cardiac tamponade due to disease progression (Fig. 2B). Then, a pericardial paracentesis was performed, and the specimens obtained from pericardial fluid showed poorly differentiated cells with a high nuclear-to-cytoplasmic ratio. With the immunohistochemical staining, neuroendocrine markers such as synaptophysin, NCAM and chromogranin were positive (Fig. 3). Besides, the additional blood tests indicated that neuron specific enolase (NSE) was $39.4 \mathrm{ng} / \mathrm{ml}$. Based on these results, SCLC transformation was confirmed. Additionally, the molecular analysis of the obtained specimens showed that the exon 19 deletion was still positive despite negative T790M mutation by cobas EGFR Mutation test v2. After pericardial paracentesis (Fig. 2C), a combination therapy of carboplatin and etoposide was administered in September 2018. After the 1st course of this regimen, mediastinal tumor and pericardial effusion were dramatically improved with a substantial decrease of NSE level, and therefore 4 cycles of this regimen were completed (Fig. 2D). The disease related SCLC transformation was considered to remain stable with normal level of NSE in March 2019.

\section{Discussion}

We herein report the case of SCLC transformation diagnosed by pericardial effusion after a long-term treatment with EGFR-TKIs including osimertinib. All previously reported cases of SCLC transformation have been diagnosed by pathological tissues of primary lesion or metastatic lesions, not pericardial effusion. Our results could provide the following two clinical implications.

First, SCLC transformation would occur after treatment with osimertinib. Among several mechanisms of acquired resistance to EGFR-TKIs, SCLC transformation reportedly accounts for $3-14 \%$ and mostly developed as the acquired resistance to 1 st- or 2nd-generation EGFR-TKIs $(2,3)$. To date, there are limited cases of SCLC transformation after osimertinib treatment (4-8). Marcoux et al (10) reported that median total time of treatment with EGFR-TKIs at the diagnosis of SCLC transformation was 15.8 months. In our case, the duration of total osimertinib treatment was 14 months and the total interval of EGFR-TKI treatment was 22 months. Taking those reports together with the present case, SCLC transformation would occur after long-term use of EGFR-TKIs regardless of the generation of EGFR-TKIs. With regard to mechanism of SCLC transformation, there have been the following two hypotheses (11). Firstly, lung cancer consists of combined SCLC and NSCLC histology at first diagnosis, and EGFR-TKI treatment would cause a component of SCLC dominant. Secondary, type II alveolar cells, the origin of some EGFR-mutant adenocarcinomas, also have the potential to become SCLC. Lung adenocarcinoma arising from these alveolar type II cells and harboring EGFR mutations might transform to SCLC under the selective pressure of TKI therapy. In our case, we did not detect a component of SCLC histologically at first diagnosis. Of note, the molecular analysis of the cell block from pericardial effusion showed that the exon 19 deletion was still positive despite negative T790M mutation, as seen in some reports (4-6). Based on those findings, we consider that this transformed SCLC developed from a common precursor of adenocarcinoma.

Second, physicians should investigate whether SCLC transformation occurs or not, especially in NSCLC 
patients who develop pericardial effusion after long-term EGFR-TKI treatment. Generally, malignant pericardial effusion develops in $2.5 \%$ of lung cancer patients and is correlated with a poor prognosis with median survival of 74.5 days $(12,13)$. Of note, even minimal pericardial effusion is reported as an independent prognostic factor for those patients (14). Generally, EGFR-mutant NSCLC patients who develop pericardial effusion after the treatment failure with EGFR-TKIs therapy present very poor prognosis due to lack of therapeutic options. On the other hand, the median survival in patients with SCLC transformation reportedly reached 10.9 months (10). Therefore, it is crucial not to overlook SCLC transformation as a treatable entity. Importantly, in our case, despite the decrease of CEA level from 100.5 to $30.0 \mathrm{ng} / \mathrm{ml}$ during S-1 treatment, his disease paradoxically progressed with high level of NSE. In fact, some previous reports about SCLC transformation revealed that pro-gastrin releasing peptide (pro-GRP) and NSE are reported to be likely to increase $(5,6)$. Therefore, it would be extremely important to measure tumor markers of pro-GRP and NSE in NSCLC patient with pericardial effusion after long-term EGFR-TKI treatment.

In conclusion, we presented the case of SCLC transformation diagnosed by pericardial effusion after long-term EGFR-TKI treatment including osimertinib. In NSCLC patients who develop pericardial effusion after long-term EGFR-TKI therapy including osimertinib, it is important to investigate whether SCLC transformation occurs or not, and measuring tumor markers.

\section{Acknowledgements}

Not applicable.

\section{Funding}

No funding was received.

\section{Availability of data and materials}

The datasets used and/or analysed during the current study are available from the corresponding author on reasonable request.

\section{Authors' contributions}

RO and AS analysed and interpreted the data and wrote the manuscript. RO, AS, MA, YS, SI, TB, SK, EH and TO evaluated the patient and participated in the therapy. KO evaluated the pathological specimens. All authors read and approved the final manuscript.

\section{Ethics approval and consent to participate}

Not applicable.

\section{Patient consent for publication}

The patient provided written informed consent for the publication of the case details and any associated images.

\section{Competing interests}

The authors declare that they have no competing interests.

\section{References}

1. Mok TS, Wu YL, Thongprasert S, Yang CH, Chu DT, Saijo N, Sunpaweravong P, Han B, Margono B, Ichinose Y, et al: Gefitinib or carboplatin-paclitaxel in pulmonary adenocarcinoma. N Engl J Med 361: 947-957, 2009

2. Yu HA, Arcila ME, Rekhtman N, Sima CS, Zakowski MF, Pao W, Kris MG, Miller VA, Ladanyi M and Riely GJ: Analysis of tumor specimens at the time of acquired resistance to EGFR-TKI therapy in 155 patients with EGFR-mutant lung cancers. Clin Cancer Res 19: 2240-2247, 2013.

3. Sequist LV, Waltman BA, Dias-Santagata D, Digumarthy S, Turke AB, Fidias P, Bergethon K, Shaw AT, Gettinger S, Cosper AK, et al: Genotypic and histological evolution of lung cancers acquiring resistance to EGFR inhibitors. Sci Trans1 Med 3: 75ra26, 2011.

4. Minari R, Bordi P, Del Re M, Facchinetti F, Mazzoni F, Barbieri F, Camerini A, Comin CE, Gnetti L, Azzoni C, et al: Primary resistance to osimertinib due to SCLC transformation: Issue of T790M determination on liquid re-biopsy. Lung Cancer 115: 21-27, 2018.

5. Iijima Y, Hirotsu Y, Mochizuki H, Amemiya K, Oyama T, Uchida Y, Kobayashi Y, Tsutsui T, Kakizaki Y, Miyashita Y and Omata M: Dynamic changes and drug-induced selection of resistant clones in a patient with EGFR-mutated adenocarcinoma that acquired T790M mutation and transformed to small-cell lung cancer. Clin Lung Cancer 19: e843-e847, 2018.

6. Taniguchi Y, Horiuchi H, Morikawa T and Usui K: Small-cell carcinoma transformation of pulmonary adenocarcinoma after osimertinib treatment: A case report. Case Rep Oncol 11: 323-329, 2018.

7. Kim TM, Song A, Kim DW, Kim S, Ahn YO, Keam B, Jeon YK, Lee SH, Chung DH and Heo DS: Mechanisms of acquired resistance to AZD9291: A mutation-selective, irreversible EGFR inhibitor. J Thorac Oncol 10: 1736-1744, 2015.

8. Ham JS, Kim S, Kim HK, Byeon S, Sun JM, Lee SH, Ahn JS, Park K, Choi YL, Han J, et al: Two cases of small cell lung cancer transformation from EGFR mutant adenocarcinoma during AZD9291 treatment. J Thorac Oncol 11: e1-e4, 2016.

9. Soria JC, Ohe Y, Vansteenkiste J, Reungwetwattana T, Chewaskulyong B, Lee KH, Dechaphunkul A, Imamura F, Nogami N, Kurata T, et al: Osimertinib in untreated EGFR-mutated advanced non-small-cell lung cancer. N Engl J Med 378: 113-125, 2018.

10. Marcoux N, Gettinger SN, O'Kane G, Arbour KC, Neal JW, Husain H, Evans TL, Brahmer JR, Muzikansky A, Bonomi PD, et al: EGFR-mutant adenocarcinomas that transform to small-cell lung cancer and other neuroendocrine carcinomas: Clinical outcomes. J Clin Oncol 37: 278-285, 2019.

11. Oser MG, Niederst MJ, Sequist LV and Engelman JA: Transformation from non-small-cell lung cancer to small-cell lung cancer: Molecular drivers and cells of origin. Lancet Oncol 16: e165-e172, 2015.

12. Hayano M, Hokamura Y, Kimura Y, Kimura T and Tokube K: Clinical studies of 16 cases of carcinomatous pericarditis. Kokyu To Junkan 39: 683-686, 1991 (In Japanese).

13. Wang PC, Yang KY, Chao JY, Liu JM, Perng RP and Yen SH: Prognostic role of pericardial fluid cytology in cardiac tamponade associated with non-small cell lung cancer. Chest 118: 744-749, 2000.

14. Kato R, Hayashi H, Chiba Y, Tanaka K, Takeda M and Nakagawa K: Prognostic impact of minimal pericardial effusion in patients with advanced non-small-cell lung cancer. Clin Lung Cancer 18: e449-e455, 2017. 